\title{
Editorial
}

\section{Advances in Fluid Dynamics of Turbomachinery}

\author{
Jingyin Li $\mathbb{D},{ }^{1}$ Philipp Epple, ${ }^{2}$ Heuy-Dong Kim, ${ }^{3}$ and Lei Tan ${ }^{4}$ \\ ${ }^{1}$ Department of Fluid Machinery and Engineering, School of Energy and Power Engineering, Xian Jiaotong University, \\ Xi'an 710049, China \\ ${ }^{2}$ Fluid Mechanics and Turbomachinery, Coburg University of Applied Sciences and Arts, Friedrich-Streib-Strasse 2, \\ 96450 Coburg, Germany \\ ${ }^{3}$ Department of Mechanical Engineering, Andong National University, 1375 Gyeongdong-ro, Andong 760-749, Republic of Korea \\ ${ }^{4}$ Department of Energy and Power Engineering, Tsinghua University, Beijing 100084, China
}

Correspondence should be addressed to Jingyin Li; jyli@mail.xjtu.edu.cn

Received 5 June 2018; Accepted 5 June 2018; Published 2 July 2018

Copyright (C) 2018 Jingyin Li et al. This is an open access article distributed under the Creative Commons Attribution License, which permits unrestricted use, distribution, and reproduction in any medium, provided the original work is properly cited.

Turbomachinery has wide applications in petrochemical industry, power engineering, ventilation, and many other fields. The new application and demand for high performance turbomachinery are always the motivations for the development in analyzing and understanding of the complicated internal flows, pressure distributions, and so on. Rapid progress has been made in recent years in efficient and reliable numerical simulation methods and new transducers and experimental methods, and new types of turbomachinery are manufactured and applied.

This special issue focuses on the advances in fluid dynamics of turbomachinery. The themes include numerical simulation, theoretical model, experimental measurement, optimal design, and blade crack detection, as well as various turbomachinery applications. From 10 submissions, 7 papers are published in this special issue. Each paper was reviewed by at least two reviewers and revised according to review comments. The papers cover the following aspects: pressure measurement, steady and unsteady numerical simulations, thermal model of internally cool, optimization design, crack detection, and rotor-bearing system.

High-pressure-ratio centrifugal compressors have been widely used in turbochargers and turboshaft engines because of their compact size, high efficiency, and wide operating range. Compared to the impeller flow with subsonic inlet conditions, the flow in transonic impeller is more complex due to the presence of shock wave. F. Lou et al. investigated the aerodynamics of a transonic impeller by using static pressure measurements and found that shock waves emerging upstream of the impeller leading edge were observed from $90 \%$ to $100 \%$ corrected speed. The shock waves moved towards the impeller trailing edge as the inlet tip relative Mach number increases.

There have been already experimental investigations on the wall pressure distributions, but the pressure measurement on the blade in the rotating system is rare. S. Münsterjohann and S. Becker integrated the miniature pressure sensors into the impeller to ensure flush mounted membranes of the sensors and avoid an impact on the flow field. The results show the unsteady pressure distribution as well as its phaselocked ensemble average and provide a deeper understanding of the flow phenomena inside channel blowers and the theory of pressure generation.

Computational fluid dynamics (CFD) is a powerful tool for the global performance prediction of turbomachinery, and steady and unsteady numerical simulations are both widely used at different time costs and with different calculation accuracies. A. Ortolan et al. compared the steady and unsteady numerical simulations for a conventional fan operating at load-controlled windmill. Numerical analysis demonstrates that the global performances obtained with the time-averaged solution of the unsteady simulation are not far different from the steady case.

The internal cooling manner for high-pressure turbine blade is crucial for the gas turbine engine service life, and the creation of blade with effective cooling system is a complicated process. I. Shevchenko et al. proposed a method of calorimetric measurement in a molten metal thermostat for the verification of a thermal model of cooled blade. This method allows obtaining local value of heat flux in each 
point of blade surface in a single experiment, and the error of determination of local heat transfer coefficients does not exceed $8 \%$ for blades with radial channels.

Performance optimization has been a long-standing challenge for the design of turbomachinery. J.-W. Kim et al. optimized the pump and turbine mode efficiencies simultaneously by using a radial basis neural network surrogate model based on the objective function values of the generated twelve design points. They successfully increased the efficiencies of both the pump and turbine modes at overall range of flow rate, except for the turbine mode at the low flow rate, compared to the reference design.

Crack failures often occur in fan blades, which result in the interruption of operation and increase maintenances, decrease product quality, and affect the safety of operators. $\mathrm{M}$. Yu et al. developed a method to detect damage based on a discrete mathematical model for fan blades by using changes in natural frequencies. This study provides an assist in investigating the effect of a crack from a different perspective.

Turboexpander is an indispensable component in energy conversion system with high speed, and its smooth operation depends on the stability of the rotor-bearing system. T. Lai et al. developed a foil bearing supporting technology by using both protuberant foil journal and thrust bearings for the turboexpander. Due to compliance of the foils in the bearing, the rotor supported by protuberant foil journal bearings could operate under over-nominal bearing clearances.

These papers represent an exciting, insightful observation into the state of the art, as well as emerging future topics, in this important interdisciplinary field. We hope that this special issue would attract a major attention of the peers.

Jingyin Li

Philipp Epple

Heuy-Dong Kim

Lei Tan 


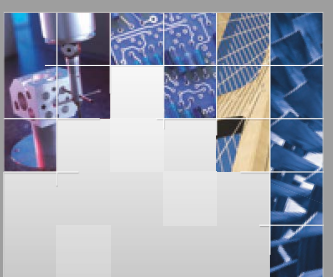

\section{Enfincering}
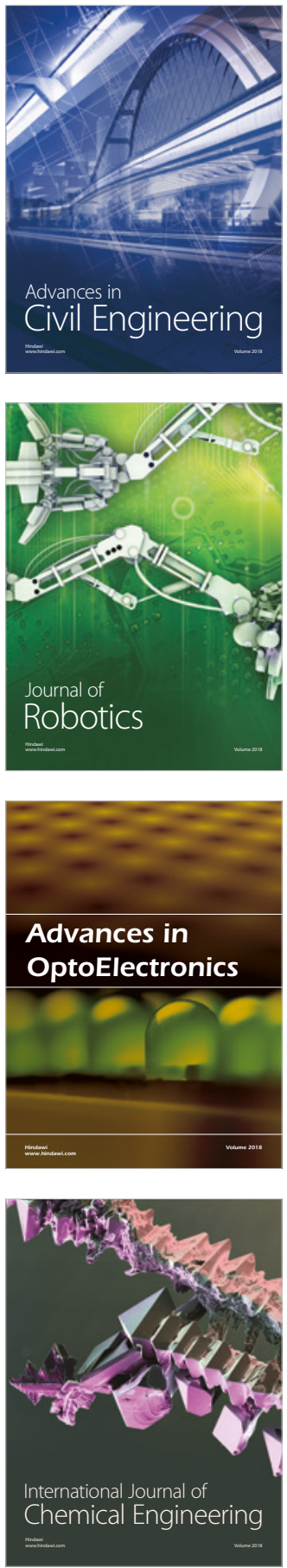

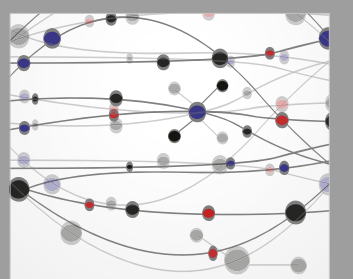

\section{Rotating \\ Machinery}

The Scientific World Journal

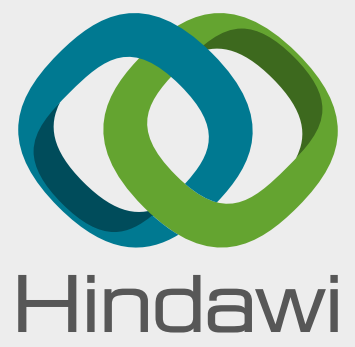

Submit your manuscripts at

www.hindawi.com
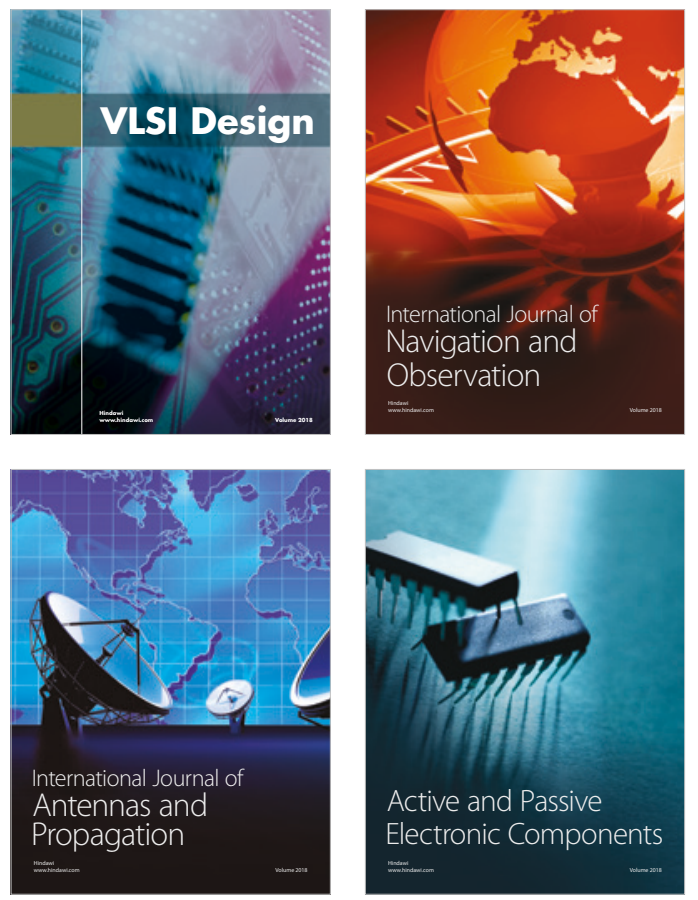
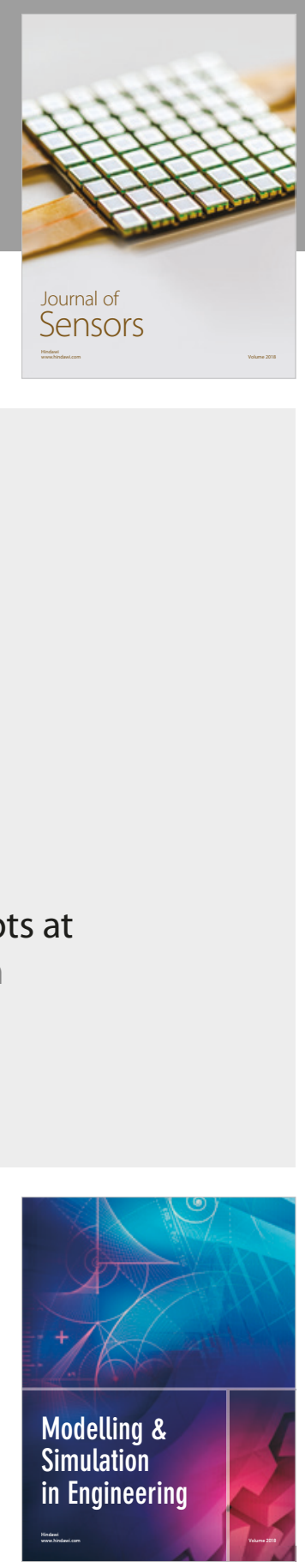

\section{Advances \\ Multimedia}
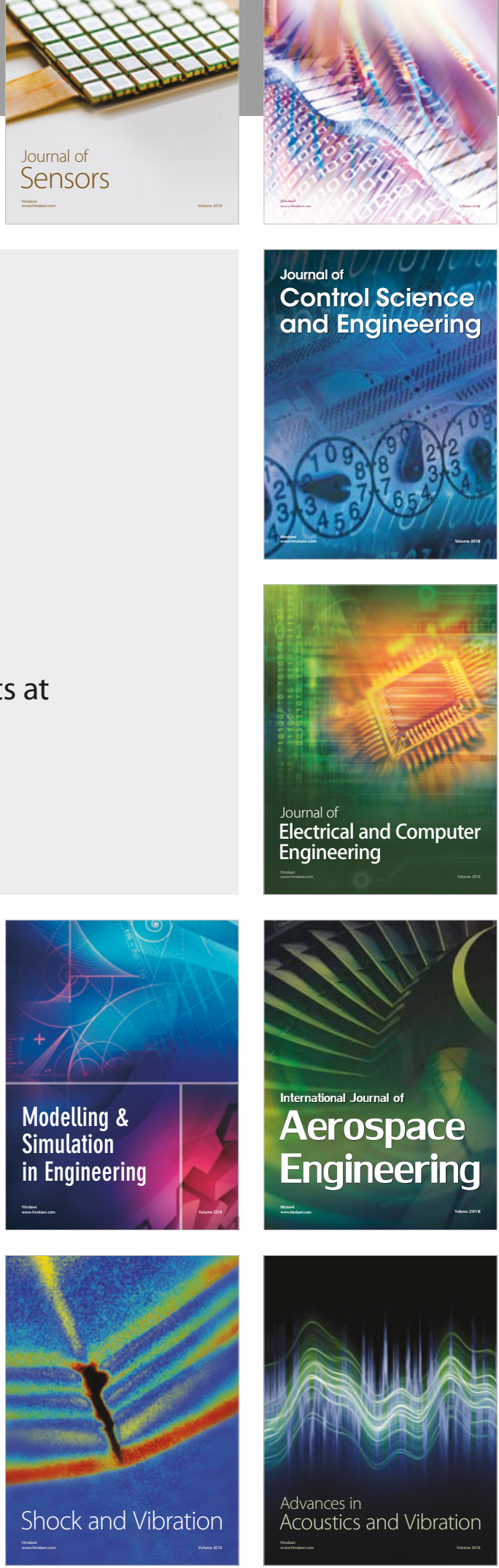